\title{
Gastos com planos de saúde das famílias brasileiras: estudo descritivo com dados das Pesquisas de Orçamentos Familiares 2002-2003 e 2008-2009
}

\author{
Spending on private health insurance plans of Brazilian families: \\ a descriptive study with data from the Family Budget Surveys \\ 2002-2003 and 2008-2009
}

\author{
Leila Posenato Garcia ${ }^{1}$ \\ Carlos Octávio Ocké-Reis ${ }^{2}$ \\ Luís Carlos Garcia de Magalhães ${ }^{3}$ \\ Ana Claudia Sant'Anna ${ }^{4}$ \\ Lúcia Rolim Santana de Freitas ${ }^{5}$
}

${ }^{1}$ Assessoria Técnica da Presidência, Instituto de Pesquisa Econômica Aplicada.SBS Bloco J/Ed. Ipea/BNDES 9o. 70076-900 Brasília DF Brasil.

leila.garcia@ipea.gov.br

${ }^{2}$ Diretoria de Estudos e Políticas do Estado, das Instituições e da Democracia, IPEA.

${ }^{3}$ Instituto de Pesquisa Econômica Aplicada, cedido para Câmara dos Deputados Federal.

${ }^{4}$ Kansas State University. ${ }^{5}$ Programa de PósGraduação em Medicina Tropical, Faculdade de Medicina, Universidade de Brasília.

\begin{abstract}
Spending on health insurance represents an important share of private expenditure on health in Brazil. The study aimed to describe the evolution of spending on private health insurance plans of Brazilian families, according to their income. Data from the Family Budget Surveys (POF) 2002-2003 and 2008-2009 were used. To compare the spending figures among the surveys, the Consumer Price Index (IPCA) was applied. The proportion of families with private health insurance expenses remained stable in both surveys (2002-2003 and 2008-2009), around $24 \%$. However, the household spending on health insurance plans increased. Among those families who spent money oh health insurance plans, the average spending increased from $R \$ 154.35$ to $R \$ 183.97$. The average spending on health insurance plans was greater with increasing household income, as well as portions of the family income and total expenditure committed to these expenses. Spending on health insurance is concentrated among higher-income families, for which it was the main component of total health expenditure. Key words Family, Health insurance, Economy, Health economics, Health inequalities
\end{abstract}

Resumo As despesas com planos de saúde correspondem a uma parcela importante dos gastos privados com saúde no Brasil. Este estudo teve como objetivo descrever a evolução dos gastos com planos de saúde das famílias brasileiras, segundo sua renda. Foram utilizados dados das Pesquisas de Orçamentos Familiares (POF) de 2002-2003 e 2008-2009. Para a comparação dos valores dos gastos entre as POF, foi feita correção mediante o Índice de Preços ao Consumidor Amplo (IPCA). A proporção de famílias que tiveram gastos com planos de saúde permaneceu estável, nas duas POF estudadas (2002-2003 e 2008-2009), em torno de $24 \%$. Todavia, o valor do gasto das famílias com planos de saúde aumentou. Entre aquelas que tiveram gasto com planos de saúde, o valor médio elevou-se de $R \$ 154,35$ para $R \$ 183,97$. O gasto médio com planos de saúde foi maior à medida que aumentava a renda das famílias, assim como as parcelas da renda e da despesa comprometidas com esses gastos. O gasto com planos de saúde está concentrado entre as famílias com maior renda. Para estas, os planos de saúde foram o principal componente do gasto total com saúde.

Palavras-chave Família, Seguro Saúde, Economia, Economia da Saúde, Desigualdades em Saúde 


\section{Introdução}

O Sistema Único de Saúde do Brasil (SUS), instituído por meio da Constituição Federal de 1988, tem contribuído sobremaneira para a ampliação do acesso ao cuidado com a saúde da população brasileira. Todavia, é relevante a coexistência do setor de saúde suplementar, que oferece diversos tipos de planos privados de saúde e apólices de seguros, e é beneficiado por subsídios públicos ${ }^{1}$.

Segundo o Instituto Brasileiro de Geografia e Estatística (IBGE) ${ }^{2}$, a partir de dados das Pesquisas Nacionais por Amostra de Domicílios (PNAD), o percentual de pessoas cobertas por pelo menos um plano de saúde cresceu entre 1998 (24,5\%), 2003 (24,6\%) e 2008 (26,3\%). Dados da Agência Nacional de Saúde Suplementar ${ }^{3}$ revelam que, entre 2003 e 2011, o tamanho do mercado de planos de saúde teve crescimento expressivo, com aumento de 36,4 milhões para 63,9 milhões de vínculos. No mesmo período, o faturamento do mercado de planos de saúde no Brasil quase dobrou e seu lucro líquido cresceu mais de duas vezes e meia acima da inflação, de $\mathrm{R} \$ 1,17$ bilhões para $\mathrm{R} \$ 4,92$ bilhões.

Os gastos com planos de saúde representam uma parcela relevante do gasto total com saúde no Brasil. Garcia et al. ${ }^{4}$, ao analisarem dados da Pesquisa de Orçamentos Familiares (POF) de 2008-2009, verificaram que as famílias brasileiras tiveram um gasto médio mensal de $\mathrm{R} \$ 153,81$ com saúde. Medicamentos e planos de saúde ocuparam a maior parcela destas despesas, correspondendo a $76 \%$. Entre as famílias de menor renda, os gastos com medicamentos corresponderam à maior parte do gasto com saúde. Todavia, entre as famílias de maior renda, os planos de saúde foram o principal componente deste gasto.

A composição dos gastos com saúde das famílias brasileiras reflete a situação do sistema de saúde nacional, formado por uma rede complexa de prestadores e compradores de serviços, e caracterizado por uma combinação público-privada, na qual os recursos privados são a principal fonte de financiamento. Apesar de haver distinção entre os componentes público e privado, estes estão imbricados no sistema. Dependendo de sua capacidade de acesso ou de pagamento, os usuários podem utilizar os serviços de natureza pública, privada ou suplementar1. É a renda familiar e individual que determina o acesso a planos de saúde e, consequentemente, o tamanho deste mercado ${ }^{5}$.

Os gastos privados com saúde são passíveis de dedução do valor devido do imposto sobre a renda, sem que exista teto para o abatimento. $\mathrm{O}$ volume do valor deduzido constitui-se gasto tributário, uma vez que o Estado, ao deixar de arrecadar, age como se estivesse realizando um pagamento. Ocké-Reis ${ }^{6}$ estimou que, em 2011, a renúncia decorrente do imposto sobre a renda da pessoa física (IRPF) correspondente aos gastos com planos de saúde equivaleu a $\mathrm{R} \$ 4,8$ bilhões.

De acordo com o Demonstrativo dos Gastos Tributários, da Receita Federal do Brasil (RFB), estimou-se que a projeção orçamentária do gasto tributário com saúde para o ano de 2014 seria de $\mathrm{R} \$ 23,7$ bilhões: $\mathrm{R} \$ 10,7$ milhões com despesas médicas; $\mathrm{R} \$ 4,2$ milhões com medicamentos; 3,7 milhões com assistência médica, odontológica e farmacêutica a empregados; e 2,9 milhões com hospitais filantrópicos (a rigor essa projeção não é a estimativa do gasto tributário efetivo). Os gastos privados com saúde, com destaque para os planos privados, têm sido considerados obstáculos para a universalização do SUS 8 .

Nesse contexto, torna-se relevante e oportuno o estudo dos gastos das famílias com planos de saúde. O presente estudo tem como objetivo descrever a evolução dos gastos com planos de saúde das famílias brasileiras, segundo sua renda, no período 2002-2009.

\section{Métodos}

Foi realizado estudo descritivo com microdados das POF, realizadas pelo Instituto Brasileiro de Geografia e Estatística (IBGE), em 2002-2003 e 2008-2009. Estas pesquisas fornecem informações detalhadas sobre tipos de gastos, quantidade, valor, especialidade de serviços, assim como sobre os rendimentos das famílias?.

A unidade de análise das POF são as famílias, ou unidades de consumo, definidas como morador ou conjunto de moradores que compartilham o mesmo estoque de alimentos e/ou conjunto de despesas alimentares. Quando não existem estoques, nem despesas com alimentos, a unidade de consumo é definida perante as despesas com moradia $^{10}$. Essa definição é importante, uma vez que, muitas vezes, as despesas registradas para um morador podem se referir a produtos comprados para outro morador, ou mesmo para um não residente. Assim, por exemplo, as despesas com planos de saúde para dependentes provavelmente serão registradas na conta do titular do plano.

A POF é composta por seis questionários: domicílios, despesa coletiva, caderneta de despesa coletiva, despesa individual, rendimento indivi- 
dual e avaliação das condições de vida ${ }^{11}$. Os períodos de referência considerados são distintos, conforme o tipo de despesa. Para a maioria das categorias dos gastos com saúde, incluindo planos de saúde, os dados se referem ao período de 90 dias antes da entrevista ${ }^{12}$.

No presente estudo, foram considerados exclusivamente os gastos monetários com planos de saúde (inclusive odontológicos). O Índice Nacional de Preços ao Consumidor Amplo (IPCA), do IBGE, disponibilizado no sítio eletrônico do Ipea Data (http://www.ipeadata.gov.br), foi empregado para correção dos valores, de modo a permitir comparação dos valores absolutos dos gastos calculados a partir das duas POF. A correção foi feita considerando como base a data de referência da POF 2002-2003 (15 de janeiro de 2003). Este índice foi selecionado por pertencer ao Sistema Nacional de Índices de Preços ao Consumidor (SNIPC), cuja atualização ocorre mediante a POF, bem como já haver sido utilizado em outros estudos da área ${ }^{12}$.

Para a categorização das famílias segundo sua renda, foi considerada a renda familiar mensal per capita total (monetária e não monetária). As famílias foram divididas inicialmente em quartos (1Q a 4Q). Por haver sido verificada importante heterogeneidade nos valores do gasto com planos de saúde entre as famílias pertencentes ao último quarto (4Q), estas foram adicionalmente divididas, destacando-se aquelas abaixo (4Qa) e acima (4Qb) do percentil 95 (5\% mais ricos). A categorização final da renda familiar mensal per capita segundo intervalos entre percentis foi: $\mathrm{p} 0$ p25 (1Q), p26-p50 (2Q), p51-p75 (3Q), p76-p95 (4Qa) e p96-p100 (4Qb).

Para o total das famílias brasileiras, e segundo estas categorias de renda, foram calculados os percentuais de famílias que tiveram gastos positivos e não nulos com planos de saúde. Os valores dos gastos, para o total das famílias, e para aquelas que tiveram gastos positivos, foram descritos por meio de medidas de tendência central e dispersão (média, mediana, primeiro quartil - p25, terceiro quartil - p75). A participação do gasto com planos de saúde em relação à renda total, à despesa total e ao gasto total com saúde foi calculada como porcentagem, para o total das famílias. Também foi calculada a variação percentual nos gastos com planos de saúde entre as POF 2002-2003 e 20082009, da seguinte maneira: (Gasto 2008-2009 Gasto 2002-2003) / Gasto 2002-2003 * 100.

Para a compatibilização dos dados das POF, utilizou-se o software SAS 9.2. As estatísticas foram estimadas por meio do software STATA 10.1, levando em conta a variável fator de expansão disponibilizada pelo IBGE. O software Excel 2010 foi empregado para produção dos gráficos.

\section{Resultados}

As amostras das POF 2002-2003 e 2008-2009 foram compostas, respectivamente, por 48.568 e 56.091 famílias. Nestas mesmas pesquisas, respectivamente 11.716 e 13.981 famílias tiveram registro de gasto com planos de saúde, o que equivale a $24,1 \%$ e $24,9 \%$. A proporção de famílias que tiveram esse tipo de gasto foi maior quanto mais elevada era a renda das famílias (Figura 1).

O valor do gasto também foi mais elevado entre as famílias com maior renda. Em 20082009, o gasto médio das famílias situadas acima do percentil 95 de renda foi $\mathrm{R} \$ 274,49$, enquanto entre aquelas do primeiro quarto (até percentil 25 ) foi $R \$ 3,15$, uma diferença relativa de 87 vezes. Segundo as POF 2002-2003 e 2008-2009, os gastos médios com planos de saúde das famílias que tiveram esse tipo de gasto foram, respectivamente, R\$ 196,12 e R\$224,73. A mediana também apresentou acréscimo, de $\mathrm{R} \$ 80,91$ para $\mathrm{R} \$$ 101,00 (Tabela 1).

No período estudado, houve variação positiva de $23 \%$ no gasto com planos de saúde para o total das famílias brasileiras e de 19\% para aquelas que tiveram gasto com planos de saúde. Houve variação positiva nos valores destes gastos em todos os grupos de renda estudados, à exceção do segundo quarto (p26-p50), para o qual foi observada relativa estabilidade. $\mathrm{O}$ maior aumento foi observado no primeiro quarto de renda ( $\mathrm{p} 0$ p25), equivalente a $66 \%$ entre as famílias que tiveram gasto com planos de saúde e $110 \%$ entre o total das famílias (Tabela 1).

A participação dos gastos com planos de saúde em relação à renda média foi pouco superior entre as famílias pertencentes ao terceiro quarto (p76-p95) em relação aos demais grupos de renda. Observou-se relativa estabilidade nesta participação, para todos os grupos de renda, entre as POF 2002-2003 e 2008-2009. Discreta elevação foi observada entre as famílias de maior renda (p96-p100), de 1,6\% para 2,1\% (Figura 2).

A participação dos gastos com planos de saúde em relação à despesa média das famílias teve comportamento semelhante àquele da participação em relação à renda, apresentando estabilidade entre as POF 2002-2003 e 2008-2009 (Figura 3).

Os gastos com planos de saúde ocuparam maior parcela dos gastos com saúde entre as fa- 


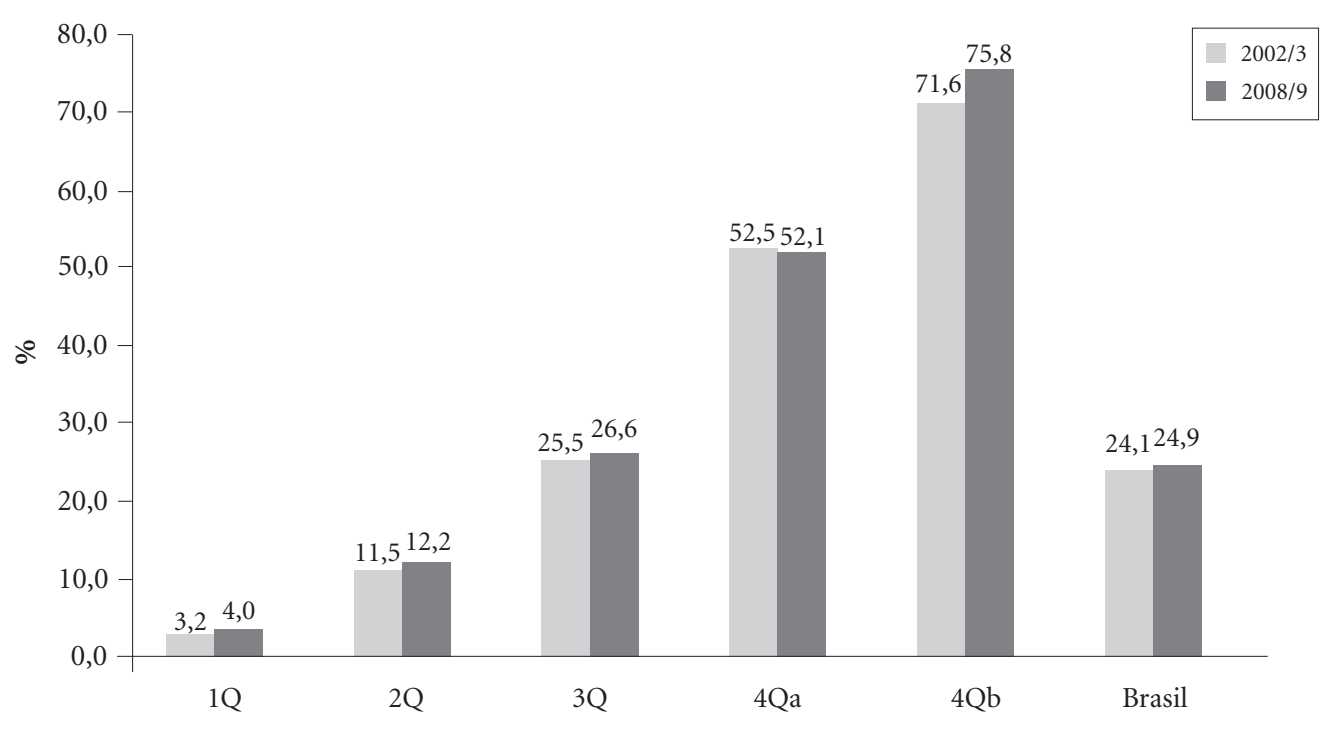

Figura 1. Proporção (\%) das famílias que tiveram gasto com planos de saúde no período de referência das Pesquisas de Orçamentos Familiares (POF) 2002-2003 e 2008-2009, segundo grupos de renda e total das famílias.

Tabela 1. Estatísticas descritivas dos valores dos gastos com planos de saúde (em reais corrigidos*) e variação percentual dos valores médios (var \%) para o total das famílias e para aquelas que tiveram gasto com planos de saúde no período de referência das Pesquisas de Orçamentos Familiares (POF) 2002-2003 e 2008-2009, segundo grupos de renda e total das famílias.

\begin{tabular}{|c|c|c|c|c|c|c|c|c|c|}
\hline \multirow{2}{*}{ Grupos de renda } & \multicolumn{4}{|c|}{$2002 / 3$} & \multicolumn{4}{|c|}{$2008 / 9$} & \multirow[b]{2}{*}{ Var \% } \\
\hline & média & p25 & mediana & p75 & média & p25 & mediana & p75 & \\
\hline \multicolumn{10}{|l|}{ Total das famílias } \\
\hline $1 Q$ & 1,50 & 0,00 & 0,00 & 0,00 & 3,15 & 0,00 & 0,00 & 0,00 & $110,1 \%$ \\
\hline $2 \mathrm{Q}$ & 8,27 & 0,00 & 0,00 & 0,00 & 8,71 & 0,00 & 0,00 & 0,00 & $5,3 \%$ \\
\hline $3 Q$ & 22,89 & 0,00 & 0,00 & 3,85 & 32,91 & 0,00 & 0,00 & 9,90 & $43,8 \%$ \\
\hline $4 \mathrm{Qa}$ & 90,60 & 0,00 & 11,93 & 113,01 & 104,74 & 0,00 & 11,09 & 134,67 & $15,6 \%$ \\
\hline $4 \mathrm{Qb}$ & 219,05 & 0,00 & 108,47 & 302,09 & 274,49 & 11,31 & 148,05 & 348,14 & $25,3 \%$ \\
\hline Brasil & 37,23 & 0,00 & 0,00 & 0,00 & 45,86 & 0,00 & 0,00 & 0,00 & $23,2 \%$ \\
\hline \multicolumn{10}{|c|}{$\begin{array}{l}\text { Famílias que tiveram gasto } \\
\text { com planos de saúde }\end{array}$} \\
\hline $1 Q$ & 47,22 & 13,81 & 25,22 & 50,35 & 78,60 & 13,73 & 33,27 & 81,60 & $66,5 \%$ \\
\hline $2 \mathrm{Q}$ & 71,65 & 17,31 & 37,36 & 78,27 & 71,21 & 15,79 & 37,08 & 90,00 & $-0,6 \%$ \\
\hline $3 \mathrm{Q}$ & 89,80 & 21,75 & 51,91 & 112,39 & 123,54 & 30,26 & 71,40 & 141,10 & $37,6 \%$ \\
\hline $4 \mathrm{Qa}$ & 172,68 & 43,34 & 106,11 & 222,65 & 201,08 & 52,69 & 126,67 & 260,00 & $16,4 \%$ \\
\hline $4 \mathrm{Qb}$ & 305,93 & 89,71 & 209,72 & 420,02 & 361,94 & 113,71 & 218,83 & 452,48 & $18,3 \%$ \\
\hline Brasil & 154,35 & 31,26 & 80,91 & 196,12 & 183,97 & 39,14 & 101,00 & 224,73 & $19,2 \%$ \\
\hline
\end{tabular}

*Valores em Reais (R\$), atualizados pelo IPCA, tendo como base janeiro de 2009.

mílias com maior renda. Enquanto entre as famílias no primeiro quarto, o gasto com planos de saúde correspondeu a 4,4\% e 6,3\% dos gastos com saúde nas POF 2002-2003 e 2008-2009, res- pectivamente, entre aquelas acima do percentil 95 de renda este gasto correspondeu a $36,2 \%$ e $43,6 \%$, respectivamente (Figura 4 ). 


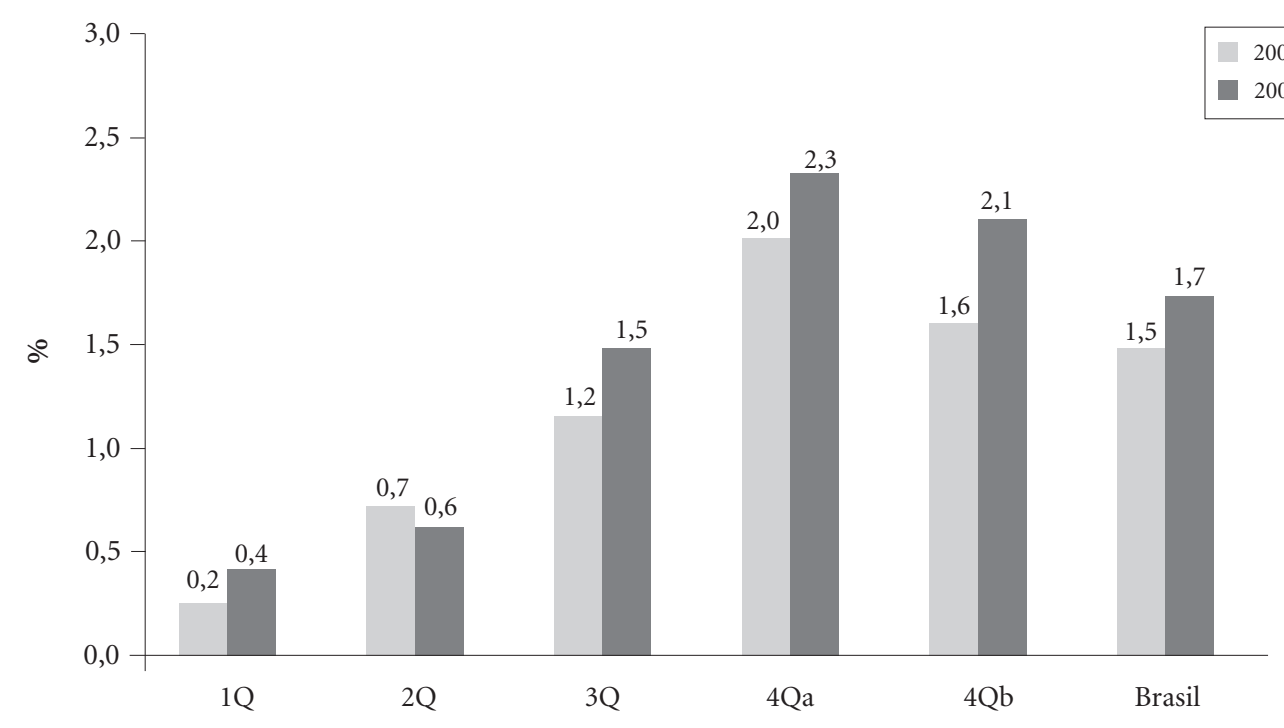

Figura 2. Participação (\%) dos gastos com planos de saúde em relação à renda média nas Pesquisas de Orçamentos Familiares (POF) 2002-2003 e 2008-2009, segundo grupos de renda e total das famílias.

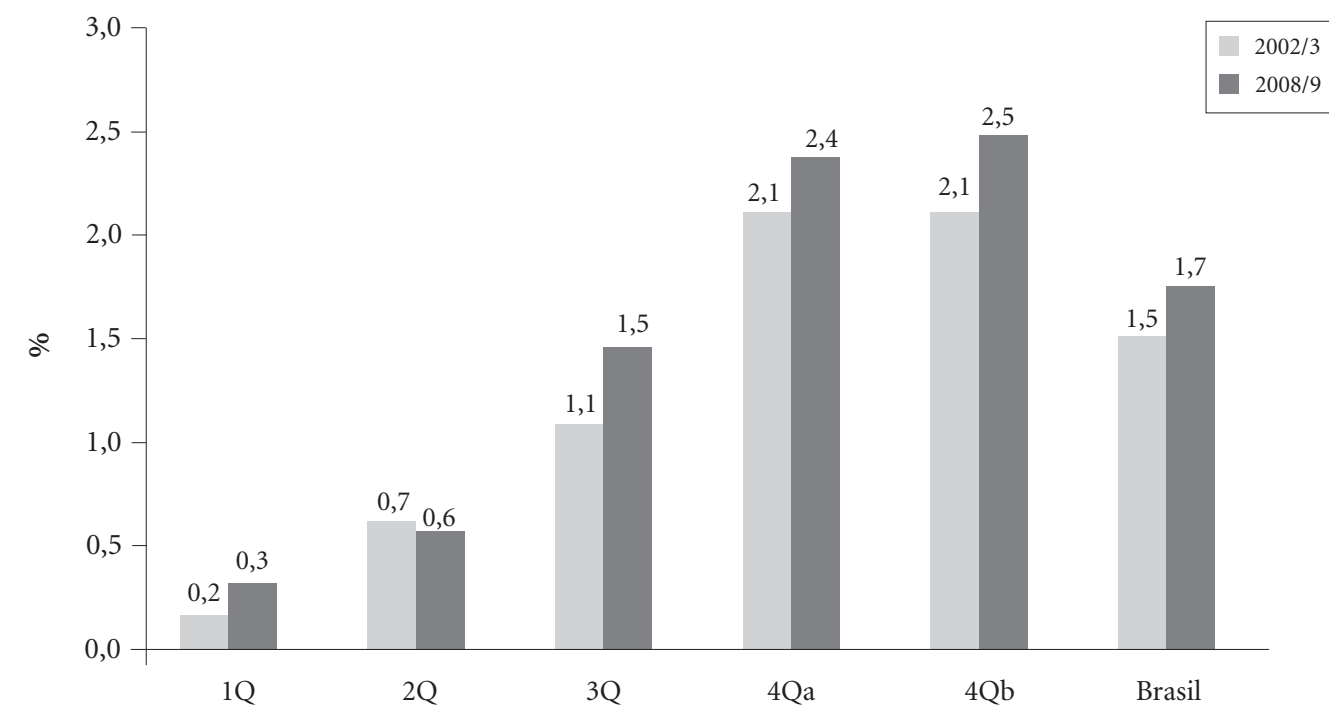

Figura 3. Participação (\%) dos gastos com planos de saúde em relação à despesa média nas Pesquisas de Orçamentos Familiares (POF) 2002-2003 e 2008-2009, segundo grupos de renda e total das famílias.

\section{Discussão}

A proporção de famílias que tiveram gastos com planos de saúde permaneceu estável, nas duas POF estudadas (2002-2003 e 2008-2009), em tor- no de $24 \%$. Todavia, o valor do gasto das famílias com planos de saúde foi crescente, assim como o comprometimento da renda das famílias com esse tipo de gasto. Verificou-se que o gasto com planos de saúde foi maior à medida que aumen- 


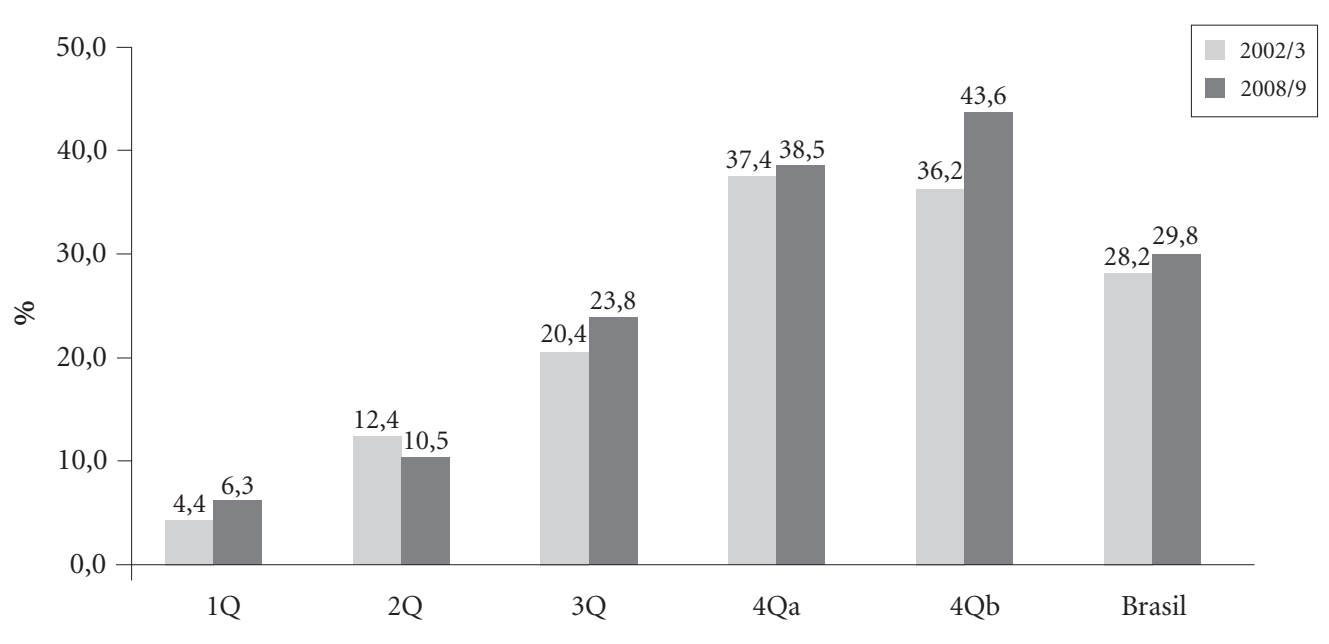

Figura 4. Participação (\%) dos gastos com planos de saúde em relação ao gasto total com saúde nas Pesquisas de Orçamentos Familiares (POF) 2002-2003 e 2008-2009, segundo grupos de renda e total das famílias.

tava a renda das famílias. A participação desse gasto em relação à renda e a despesa das famílias também foi mais elevada entre as famílias com maior renda. Estas famílias tiveram os planos de saúde como principal componente do seu gasto total com saúde.

A demanda por serviços de saúde, e também por planos de saúde privados, é determinada por diversos fatores, como necessidade sentida, fatores psicossociais, seguridade social, demografia, epidemiologia, utilização dos serviços, regulamentação e fatores culturais ${ }^{13}$. Além dos fatores relacionados à demanda, o gasto com saúde também é fortemente determinado pela renda. Outros estudos revelaram que estes gastos aumentam à medida que se eleva a renda das famílias $^{4,9,12}$.

O vínculo formal de trabalho também é um fator que pode determinar a adesão a plano de saúde. Segundo dados da PNAD 2008, das 49,2 milhões de pessoas dispunham de pelo menos um plano de saúde, $77,5 \%$ estavam vinculadas a planos de empresas privadas e $22,5 \%$ a planos de assistência a servidores públicos. Do total de pessoas cobertas por plano de saúde, 47,8\% eram titulares do plano de saúde único ou principal que possuíam. Entre os titulares dos planos de saúde, $71,3 \%$ pagavam mensalidades, sendo que $43,2 \%$ o pagavam por meio do trabalho atual ou do trabalho anterior e $28,1 \%$ o pagavam diretamente à empresa do plano de saúde. Ainda, 8,2\% tinham seus planos pagos por outro morador do domicílio ou por terceiro não morador do domi- cílio. Assim, fica evidente que as famílias arcavam com as despesas de planos de saúde de 79,5\% dos titulares. Apenas 20,5\% deles tinham seus planos pagos integralmente pelo empregador ${ }^{14}$.

O presente estudo evidenciou que não houve aumento da proporção de famílias que tiveram gasto com planos de saúde, mas houve elevação do valor médio do gasto. Estudo que verificou a composição dos gastos com saúde das famílias residentes nas diferentes regiões brasileiras, revelou que, no período compreendido entre as POF 2002-2003 e 2008-2009, a participação do gasto com planos de saúde em relação ao total do gasto com saúde das famílias brasileiras apresentou pequena variação, de $28,1 \%$ para $29,8 \%{ }^{15}$. Isso é coerente com o fato de que a receita das operadoras de planos de saúde elevou-se de $\mathrm{R} \$ 28$ bilhões, em 2003, para R\$ 73 bilhões, em $2010^{3}$. Segundo a PNAD 2008, o valor mais frequente da mensalidade dos planos de saúde foi de R\$100,00 a R\$ $200,00(14,5 \%)$, seguida pela faixa de mais de R\$ 50,00 a R\$ $100,00(13,1 \%)$, sendo que $4,5 \%$ das pessoas pagavam mais de $\mathrm{R} \$ 500,00$ pelo plano de saúde ${ }^{14}$.

Corroborando os achados do presente estudo, dados da PNAD 2008 revelam que a cobertura por plano de saúde estava positivamente relacionada ao rendimento mensal domiciliar per capita. Entre os residentes em domicílios com rendimento per capita de até $1 / 4$ do salário mínimo, apenas $2,3 \%$ tinham plano de saúde, enquanto esse percentual equivaleu a $82,5 \%$ entre aqueles residentes em domicílios cujo rendimen- 
to era superior a cinco salários mínimos. Ademais, o percentual de pessoas cobertas por planos de saúde era maior nas áreas urbanas $(29,7 \%)$ do que nas rurais $(6,4 \%)$. As Regiões Sudeste e Sul registraram coberturas $(35,6 \%$ e $30,0 \%$, respectivamente) aproximadamente três vezes maiores do que aquelas observadas no Norte $(13,3 \%)$ e Nordeste $(13,2 \%)^{14}$. Segundo a ANS ${ }^{16}$, em dezembro de 2011, a cobertura dos planos de assistência médica era mais elevada nos estados de São Paulo e Rio de Janeiro, respectivamente, 44,8\% e $37,7 \%$. É importante ressaltar que os planos de saúde contribuem para gerar desigualdades no acesso e na utilização de serviços de saúde, uma vez que cobrem um estrato privilegiado da população brasileira, que inclui pessoas com renda mais elevada, cor da pele branca, maior nível de escolaridade, inseridas em determinados ramos no mercado de trabalho e residentes em capitais ou regiões metropolitanas ${ }^{17}$. Bahia et al. ${ }^{18}$ destacam, entre as consequências da segmentação do sistema de saúde brasileiro (entre público e privado), o elevado padrão de gastos privados com saúde, a transferência de riscos relacionados com custos assistenciais do privado para o público e o padrão diferenciado, segundo condição de cobertura, de utilização de serviços, no que tange à quantidade de serviços utilizados e, possivelmente, à qualidade da atenção prestada.

Muitas vezes, as famílias com menor renda adquirem planos de saúde básicos, que não provêm acesso a determinados exames ou procedimentos, o que pode trazer consequências adversas para estes e para o SUS, quando seus usuários têm diagnóstico tardio de determinadas doenças. De acordo com a PNAD $2008^{14}$, a maioria dos planos de saúde, $88,9 \%$, dava direito a consultas, internações e exames complementares. Todavia, além das mensalidades pagas, $29,3 \%$ das pessoas realizavam pagamentos adicionais pelo serviço utilizado. O copagamento era mais frequente em planos que abrangiam apenas consultas médicas $(47,8 \%)$ e consultas médicas e exames complementares $(52,4 \%)$. Nos planos com direito a consultas médicas e internações hospitalares, o percentual de coparticipação ficou em torno de 30,0\%.

O presente estudo apresenta algumas limitações que merecem ser mencionadas. Destacam-se aquelas inerentes aos estudos realizados a partir de dados secundários e de informações autorreferidas, assim como aquelas referentes à metodologia das POF. Assim, existem tanto a possibilidade de subestimação do gasto, no caso de subregistro por parte das famílias, como de superestimação, em decorrência de se considerar apenas as famílias que tiveram registro de gasto durante o período de referência das $\mathrm{POF}^{12,19}$. Outra limitação se refere à apresentação dos valores médios do gasto e do percentual do gasto com planos de saúde em relação à renda, uma vez que é conhecida sua distribuição assimétrica, verificada por meio da discrepância entre a média e a mediana revelada nos resultados do presente estudo. Ademais, é importante destacar que os gastos com planos de saúde apresentados referem-se exclusivamente aos gastos das famílias, ou seja, não foram computados aqueles correspondentes à parcela desembolsada pelas empresas nos planos de saúde coletivos. O fato de não ser possível atribuir a totalidade dos gastos feitos pela família ao pagamento da mensalidade de planos de saúde para membros da família, também pode se constituir limitação, apesar dessa contribuição ser pequena ${ }^{10}$.

Todavia, as limitações são parcialmente minimizadas pelo cuidadoso processo de realização das POF, desde o planejamento, passando pela coleta de dados, até a revisão crítica dos resultados, que confere a estas pesquisas, valiosas fontes de dados sobre consumo das famílias brasileiras, um elevado padrão de qualidade ${ }^{10,20}$. Apesar das limitações existentes, acredita-se que o presente estudo contribuiu para agregar conhecimento a respeito da magnitude e da relevância do gasto com planos de saúde para as famílias brasileiras.

A relevância desse gasto também se reflete no gasto tributário, uma vez que não existe limite para a dedução fiscal dos gastos com planos de saúde. Ocké-Reis ${ }^{6}$ calculou, a partir de dados da Receita Federal, o valor do gasto tributário, ou renúncia fiscal em saúde, que corresponde ao imposto que o Estado deixa de recolher ao permitir que os contribuintes deduzam os gastos com planos e serviços privados de saúde do imposto de renda. Em 2011, as deduções de valores pagos aos planos de saúde atingiram R $\$ 7,7$ bilhões, aproximadamente a metade do total de R $\$ 15,8$ bilhões do gasto tributário em saúde. Naquele ano, o gasto tributário em saúde correspondeu a quase um quarto do valor total do gasto federal com saúde (R\$ 70 bilhões). No período 2003-2011, o gasto tributário associado aos planos de saúde representou, em média, $10 \%$ do faturamento total das operadoras.

No presente estudo, ficou evidente que são as famílias com maior renda que têm gastos mais elevados com planos de saúde. Assim, também são estas as famílias mais beneficiadas com a renúncia fiscal. A dedução das despesas no imposto de renda das pessoas físicas somente pode ser 
realizada por contribuintes que pagam imposto de renda e que preenchem a declaração no modelo completo. O fato de não haver limite para a dedução dos gastos com planos de saúde do valor do imposto de renda, aprofunda ainda mais as iniquidades na distribuição de recursos para a saúde.

O elevado volume do gasto tributário com planos de saúde em um contexto orçamentário no qual se considera que existe um subfinanciamento crônico do SUS é uma questão que merece reflexão. É possível que a renúncia fiscal contribua para a discrepância que existe nos gastos com saúde no Brasil: aproximadamente um quarto da população brasileira tem acesso a serviços privados de saúde e mais da metade das despesas com consumo final de bens e serviços de saúde é paga pelas famílias, apesar de existir um sistema de saúde público, universal e gratuito. Segundo dados do estudo Contas-satélite de saúde, realizado pelo IBGE, no período 2007-2009, as despesas com consumo final de bens e serviços de saúde corresponderam a $8,5 \%$ do PIB. Em 2009, as famílias gastaram $\mathrm{R} \$ 157$ bilhões e o governo, $\mathrm{R} \$ 124$ bilhões. Ou seja, as famílias arcaram com 56\% destas despesas ${ }^{21}$. Cabe esclarecer que os gastos das famílias se referem a planos e seguros privados de saúde e ao gasto direto do bolso, ou gasto "out-of -pocket", com medicamentos e outros itens, enquanto a parcela do governo engloba os gastos das três esferas (Federal, Estadual e Municipal).

A dimensão econômico-financeira é fundamental para a sustentabilidade do SUS. São reconhecidos os problemas de gestão existentes, inerentes a um sistema com um curto período de existência, descentralizado em um país com dimensões continentais, e estruturado de maneira fragmentada. Todavia, o problema do subfinanciamento é real, compromete a sustentabilidade do sistema público e impede a concretização do SUS como foi concebido, para prover atendimento universal e integral. Apesar do crescimento nos gastos públicos com saúde, de $\mathrm{R} \$ 502,36$ reais per capita em 2007, para $\mathrm{R} \$ 645,27$ per capita em 2009 (correspondente a $28,4 \%$ ), a participação das despesas com saúde no total das despesas de consumo final da administração pública permaneceu em torno de $18 \%{ }^{21}$.

O presente estudo evidenciou que, em 20082009, quase $25 \%$ das famílias brasileiras tiveram gasto com planos de saúde. É importante destacar que a expansão do mercado dos planos de saúde foi patrocinada pelo Estado, por meio de diversos mecanismos. Desde 1968, um conjunto de incentivos governamentais foi dirigido a esse mercado. Dentre os incentivos diretos, destacamse empréstimos para construção de hospitais e compra de equipamentos, além da concessão de privilégios fiscais para entidades filantrópicas. Dentre os indiretos, menciona-se a criação de normas, no plano jurídico, para a expansão do mercado de planos.

Recentemente, tem havido intenso debate sobre a existência de subsídio público ao subsistema privado de saúde ${ }^{22,23}$. Esse subsídio é altamente regressivo, ou seja, beneficia as pessoas com maior renda, que têm capacidade de pagamento, ao mesmo tempo em que reduz a capacidade de financiamento do SUS.

Atualmente, o Estado ainda financia, de forma indireta, as empresas de planos de saúde. A imposição de limites para a renúncia fiscal das despesas com planos de saúde, ou mesmo a eliminação dessa forma de renúncia poderia ser fonte importante de recursos para o SUS. É possível que, sem o aporte de subsídios, o comportamento dos contribuintes fosse modificado. Estes poderiam deixar de gastar, migrar despesa para outros gastos, ou mesmo sonegar informações sobre rendimentos tributáveis, como forma de compensar a despesa adicional resultante da redução da renúncia. Também é possível que os lucros extraordinários dos planos de saúde seriam reduzidos. Nessa situação, o mercado de planos tenderia a se reduzir, a menos que passasse a receber outra forma de subsidio estatal. Do ponto de vista da saúde pública, seria mais interessante investir este dinheiro no SUS ${ }^{23}$.

Tal medida, aparentemente radical, poderia desagradar os estratos da população que recebem o beneficio da isenção tributária. A população brasileira tende a reduzir a questão da saúde aos hospitais e equipamentos sofisticados, cujo acesso desses estratos é mediado pelos planos de saúde. Ademais, é uma visão equivocada crer que é possível construir um sistema de saúde olhando somente para a assistência. O SUS é muito maior e inclui ações mais abrangentes, como aquelas voltadas à vigilância em saúde, que têm sido fundamentais para a melhoria dos indicadores de saúde da população brasileira. Um exemplo é o Programa Nacional de Imunizações (PNI), o maior programa de imunizações do mundo, que contribuiu sobremaneira para a diminuição da morbimortalidade por doenças transmissíveis no País ${ }^{25}$.

É importante destacar, ainda, que apesar da expansão do número de beneficiários, a participação dos planos de saúde no financiamento dos atendimentos é pouco expressiva. Segundo 
dados da PNAD 2008, os planos de saúde foram responsáveis por apenas $27 \%$ dos atendimentos realizados ${ }^{25}$. Essa participação é ainda mais reduzida quando se considera os procedimentos de alto custo, que são financiados principalmente pelo SUS. Segundo a PNAD 2008, o maior protagonismo do SUS se deu nos atendimentos por químio e radioterapia, hemodiálise e hemoterapia (financiando $73,3 \%$ dos mesmos), nas consultas médicas $(62,7 \%)$ e nos exames complementares $(59,2 \%)$. Portanto, o SUS financiou não somente os atendimentos de mais baixo custo, mas também e, principalmente, aqueles mais onerosos $^{26}$. Estudo realizado no Rio de Janeiro, em 2003, revelou que as proporções de transplantes de fígado e hemodiálises remuneradas diretamente pelo SUS variaram de $89 \%$ a $96 \%{ }^{18}$. Além disso, os planos privados de saúde não atuam de maneira sinérgica com o sistema público. Segundo Ocké-Reis ${ }^{26}$, o mercado privado oferece cobertura duplicada aos serviços oferecidos pelo SUS. É conhecido que usuários que necessitam de procedimentos complexos não cobertos pelos planos de saúde recorrem ao SUS. Apesar de beneficiários dos planos usufruírem dos serviços públicos, as operadoras, em grande parte, não realizam de maneira adequada o ressarcimento das despesas de seus beneficiários para o governo. Ainda, apesar dos lucros crescentes e dos subsídios do governo, as reclamações de usuários de planos de saúde não param de aumentar. Pesquisa encomendada pela Associação Paulista de Medicina ao Datafolha, realizada em $2013^{27}$, junto à população adulta do estado de São Paulo que havia utilizado serviços dos planos de saúde nos 24 meses anteriores à entrevista, revelou grande insatisfação por parte dos usuários. Problemas foram relatados por $79 \%$ dos entrevistados, com destaque para a demora na marcação dos atendimentos $(52 \%)$, a saída de médicos $(28 \%)$ e a demora na autorização de consultas (25\%). Três em cada dez pessoas que tinham planos de saúde recorreram ao SUS ou ao atendimento particular para receber atendimento nos últimos dois anos. Pesquisa anterior do Datafolha junto a profissionais de medicina, revelou que 9 em cada 10 relataram insatisfação por problemas diversos, como as pressões exercidas pelos planos para a redução na solicitação de exames, além de interferências até mesmo em internações ${ }^{27}$.

Ao estudar os possíveis efeitos dos seguros privados sobre os sistemas nacionais de saúde, Santos ${ }^{28}$ concluiu que a cobertura duplicada pode contribuir para a iniquidade na oferta, no acesso e no uso dos serviços, assim como incentivar o desenvolvimento da atuação do setor privado nos serviços em que a população tem dificuldade de acesso no sistema público, como consultas especializadas, cirurgias eletivas, e medicamentos.

A despeito das questões apontadas, os interesses do mercado privado na saúde ainda se sobressaem. Entre as polêmicas atuais, destacam-se propostas para a criação de planos de saúde especiais para aposentados, uma vez que a maioria dos planos são coletivos e os beneficiários passam a arcar com a totalidade do valor quando se aposentam; planos de saúde de baixo custo para segmentos da população com menor renda e para empregadas domésticas. Em outubro de 2013, foi anunciado que uma proposta para abertura de uma linha de crédito do Banco Nacional de Desenvolvimento Econômico e Social (BNDES), para financiar empresas de planos de saúde, estava sendo estudada pelo governo federal ${ }^{29}$.

Acredita-se que essas propostas estão na contramão das evidências que indicam a necessidade de maior financiamento para o SUS e de fortalecer este sistema público, criado para garantir o direito à saúde, na Constituição Federal de 1988. Ademais, a criação de novas modalidades de planos de saúde possivelmente não contribuiria para reduzir a pressão da demanda por serviços do sistema público e, tampouco, no financiamento desse sistema. Os planos de saúde também não contribuem para a consecução dos objetivos gerais do SUS - universalidade, integralidade e equidade - nem de objetivos sociais, como a melhoria das condições de vida da população. Segundo Santos (2001), os planos de saúde "corroem" esses objetivos ${ }^{28}$.

Inúmeros são os desafios na busca da construção de políticas tributárias e de saúde que possam enfrentar essas questões. $\mathrm{O}$ estudo dos gastos privados das famílias com saúde, incluindo os gastos com planos de saúde pode fornecer elementos para o melhor conhecimento do financiamento privado em saúde no Brasil, bem como proporcionar subsídios para a elaboração de propostas que visem contribuir para o alcance dos objetivos do SUS.

\section{Colaboradores}

LP Garcia, CO Ocké-Reis, LCG Magalhães, AC Sant'Anna e LRS Freitas participaram igualmente de todas as etapas de elaboração do artigo. 


\section{Referências}

1. Paim J, Travassos C, Almeida C, Bahia L, Macinko J. The Brazilian health system: history, advances, and challenges. The Lancet 2011; 377(9779):1778-1797.

2. Instituto Brasileiro de Geografia e Estatística (IBGE). Indicadores sociodemográficos e de saúde no Brasil 2009. Rio de Janeiro: IBGE; 2009.

3. Brasil. Agência Nacional de Saúde Suplementar (ANS). Caderno de Informação da Saúde Suplementar: beneficiários, operadoras, planos de saúde. Rio de Janeiro: ANS; 2011.

4. Garcia LP, Sant'Anna AC, Magalhães LCG, Aurea AP. Gastos com saúde das famílias brasileiras residentes em regiões metropolitanas: composição e evolução no período 1995-2009. Cien Saude Colet 2013; 18(1):115128.

5. Bahia L. As contradições entre o SUS universal e as transferências de recursos públicos para os planos e seguros privados de saúde. Cien Saude Colet 2008; 13(5):1385-1397.

6. Ocké-Reis CO. Mensuração dos gastos tributários: o caso dos planos de saúde 2013. [acessado 2013 mar 31]. Disponível em: http://www.ipea.gov.br/portal/images/stories/PDFs/nota_tecnica/130528_notatecnicadiest05. pdf

7. Silva AC, Gama FN, Machado MAM. Demonstrativo dos Gastos Governamentais Indiretos de Natureza Tributaria (Gastos Tributarios) - PLOA 2014. (Receita Federal, 2014). [acessado 2014 set 24].Disponível em: http:// www.receita.fazenda.gov.br/publico/estudotributario/ BensTributarios/2014/DGT2014.pdf

8. Bahia L. Avanços e percalços do SUS: a regulação entre o público e o privado. Trabalho, educação e saúde 2006; 4(1):159-169.

9. Andrade, M V, Lisboa, M B. Determinantes dos gastos pessoais privados com saúde no Brasil. In: Silveira FG, Servo LM, Menezes T, Piola SF, organizadores. Gasto e consumo das famílias brasileiras contemporâneas. Brasília: Ipea; 2006. p. 84-106.

10. Instituto Brasileiro de Geografia e Estatística (IBGE). Pesquisa de orçamentos familiares 2008-2009: despesas, rendimento e condições de vida. Rio de Janeiro: IBGE; 2010.

11. Menezes T, Campolina B, Silveira FG, Servo LMS, Piola SF. O gasto e a demanda das famílias em saúde: uma análise a partir da POF de 2002-2003. In: Silveira FG, Servo LM, Menezes T, Piola SF, organizadores. Gasto e consumo das famílias brasileiras contemporâneas. Brasília: Ipea; 2006. p. 313-344.

12. Silveira FG, Osorio RG, Piola SF. Os gastos das famílias com saúde. In: Silveira FG, Servo LM, Menezes T, Piola SF, organizadores. Gasto e consumo das famílias brasileiras contemporâneas. Brasília: Ipea; 2006. p. 107-124.

13. Zucchi P, Del Nero C, Malik AM. Gastos em saúde: os fatores que agem na demanda e na oferta dos serviços de saúde. Saúde e Soc 2000; 9(1-2):127-150.

14. Instituto Brasileiro de Geografia e Estatística (IBGE). Pesquisa Nacional por Amostra de Domicílios: Um Panorama da Saúde no Brasil, Acesso e utilização dos serviços, condições de saúde e fatores de risco e proteção à saúde 2008. Rio de Janeiro: IBGE; 2010.
15. Garcia LP, Sant'Anna AC, Freitas LRS, Magalhães LCG. Gastos com saúde das famílias brasileiras: um recorte regional a partir das Pesquisas de Orçamentos Familiares 2002-2003 e 2008-2009. In: Monastério LM, Neri MC, Soares SSD, organizadores. Brasil em Desenvolvimento. Brasília: Ipea; 2013.

16. Brasil. Agência Nacional de Saúde Suplementar (ANS). Foco: saúde suplementar. Rio de Janeiro: ANS; 2012.

17. Pinto LF, Soranz DR. Planos privados de assistência à saúde: cobertura populacional no Brasil. Cien Saude Colet 2004; 9(1):85-98.

18. Bahia L, Simmer E, Oliveira DC. Cobertura de planos privados de saúde e doenças crônicas: notas sobre utilização de procedimentos de alto custo. Cien Saude Colet 2004; 9(4):921-929.

19. Barros AJ, Bertoldi AD. Out-of-pocket health expenditure in a population covered by the Family Health Program in Brazil. Int J Epidemiol 2008; 37(4):758-765.

20. Diniz BPC, Servo LMS, Piola SF, Eirado M. Gasto das famílias com saúde no Brasil: evolução e debate sobre gasto catastrófico. In: Silveira FG, Servo LM, Menezes T, Piola SF, organizadores. Gasto e consumo das famílias brasileiras contemporâneas. Brasília: Ipea; 2007. p. 143-166.

21. Instituto Brasileiro de Geografia e Estatística (IBGE). Coordenação de Contas Nacionais. Conta-satélite de saúde: Brasil: 2007-2009. Rio de Janeiro: IBGE; 2012.

22. Bahia L. O sistema de saúde brasileiro entre normas e fatos: universalização mitigada e estratificação subsidiada. Cien Saude Colet 2009; 14(3):753-762.

23. Piola SF. Tendências do financiamento da Saúde. Debates GV Saúde 2006; 2:12-14.

24. Domingues CMAS, Teixeira AMS. Coberturas vacinais e doenças imunopreveníveis no Brasil no período 1982-2012: avanços e desafios do Programa Nacional de Imunizações. Epidemiol E Serviços Saúde 2013; 22(1):9-27.

25. Porto SM, Santos IS, Ugá MAD. A utilização de serviços de saúde por sistema de financiamento. Cien Saude Colet 2006; 11(4):895-910.

26. Ocké-Reis CO. SUS: o desafio de ser único. Rio de Janeiro: Fiocruz; 2012.

27. Usuários de planos de saúde recorrem ao SUS para receber atendimento, diz pesquisa. Folha de São Paulo. São Paulo; 2013; [acessado 2013 out 17]. Disponível em: http://www1.folha.uol.com.br/cotidiano/2013/10/ 1358002-usuarios-de-planos-de-saude-recorrem-aosus-para-receber-atendimento.shtml

28. Santos IS. Evidência sobre o mix público-privado em países com cobertura duplicada: agravamento das iniquidades e da segmentação em sistemas nacionais de saúde. Cien Saude Colet 2011; 16(6):2743-2752.

29. Formenti L, Matais A. BNDES deve abrir linha de crédito para financiar operadoras de plano de saúde. O Estado de São Paulo. São Paulo; 2013; [acessado 2013 out 24] Disponível em: http://saude.estadao.com.br/noticias/ geral,bndes-deve-abrir-linha-de-credito-para-financiar -operadoras-de-plano-de-saude, 1088909

Artigo apresentado em 16/06/2014

Aprovado em 29/09/2014

Versão final apresentada em 01/10/2014 